\title{
Extreme Lower Previsions and Minkowski Indecomposability
}

Jasper De Bock \& Gert de Cooman

\author{
ECSQARU 2013, Utrecht
}




\section{Bishop-De Leeuw theorem}

Let $V$ be a locally convex Hausdorff topological linear space, and let $C$ be a non-empty convex and compact subset of $V$. Denote by $A(C)$ the linear space of all continuous affine real maps $a$ on $C$. Then for every $c \in C$ there exists a $\sigma$-additive probability measure $\mu_{c}$ supported on the set $\operatorname{ext}(C)$ of extreme points of $C$ such that

$$
a(c)=\int_{\operatorname{ext}(C)} a(e) \mathrm{d} \mu_{c}(e) \text { for all } a \in A(C) .
$$




\section{Bishop-De Leeuw theorem}

Let $V$ be a locally convex Hausdorff topological linear space, and let $C$ be a non-empty convex and compact subset of $V$. Denote by $A(C)$ the linear space of all continuous affine real maps $a$ on $C$. Then for every $c \in C$ there exists a $\sigma$-additive probability measure $\mu_{c}$ supported on the set $\operatorname{ext}(C)$ of extreme points of $C$ such that

$$
a(c)=\int_{\operatorname{ext}(C)} a(e) \mathrm{d} \mu_{c}(e) \text { for all } a \in A(C) .
$$
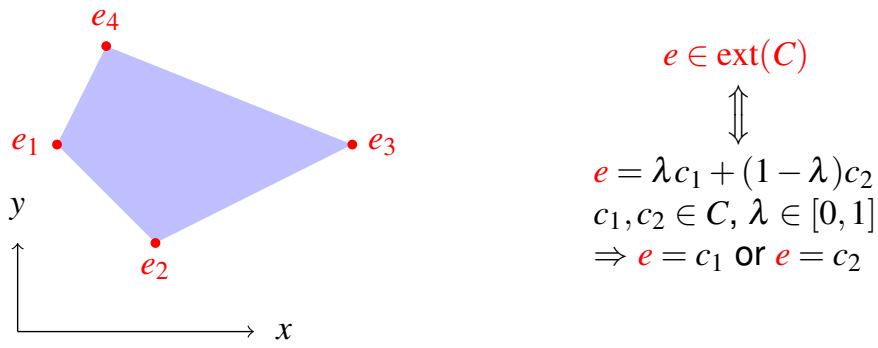


\section{Bishop-De Leeuw theorem}

Let $V$ be a locally convex Hausdorff topological linear space, and let $C$ be a non-empty convex and compact subset of $V$. Denote by $A(C)$ the linear space of all continuous affine real maps $a$ on $C$. Then for every $c \in C$ there exists a $\sigma$-additive probability measure $\mu_{c}$ supported on the set $\operatorname{ext}(C)$ of extreme points of $C$ such that

$$
a(c)=\int_{\operatorname{ext}(C)} a(e) \mathrm{d} \mu_{c}(e) \text { for all } a \in A(C) .
$$

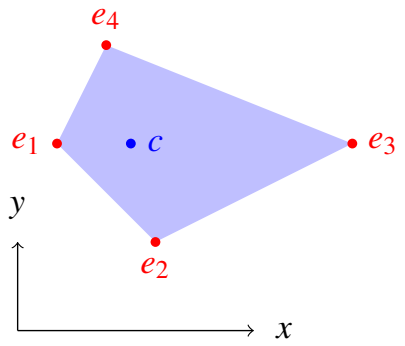

$$
\begin{aligned}
a(c)=\sum_{i=1}^{4} a\left(e_{i}\right) \mu_{c}\left(\left\{e_{i}\right\}\right) \text { for all } a \in A(C) \\
\begin{aligned}
a_{x}(c):=c(x) \Longrightarrow c(x) & =\sum_{i=1}^{4} e_{i}(x) \mu_{c}\left(\left\{e_{i}\right\}\right) \\
a_{y}(c):=c(y) \Longrightarrow c(y) & =\sum_{i=1}^{4} e_{i}(y) \mu_{c}\left(\left\{e_{i}\right\}\right) \\
c & =\sum_{i=1}^{4} e_{i} \mu_{c}\left(\left\{e_{i}\right\}\right)
\end{aligned}
\end{aligned}
$$




\section{Bishop-De Leeuw theorem}

Let $V$ be a locally convex Hausdorff topological linear space, and let $C$ be a non-empty convex and compact subset of $V$. Denote by $A(C)$ the linear space of all continuous affine real maps $a$ on $C$. Then for every $c \in C$ there exists a $\sigma$-additive probability measure $\mu_{c}$ supported on the set $\operatorname{ext}(C)$ of extreme points of $C$ such that

$$
a(c)=\int_{\operatorname{ext}(C)} a(e) \mathrm{d} \mu_{c}(e) \text { for all } a \in A(C) .
$$

This trick keeps working for more general cases!

If $C$ consists of real-valued functions $c$ on some space $\Omega$

$$
c(\omega)=\int_{\operatorname{ext}(C)} e(\omega) \mathrm{d} \mu_{c}(e) \text { for all } \omega \in \Omega .
$$




\section{Bishop-De Leeuw theorem}

Let $V$ be a locally convex Hausdorff topological linear space, and let $C$ be a non-empty convex and compact subset of $V$. Denote by $A(C)$ the linear space of all continuous affine real maps $a$ on $C$. Then for every $c \in C$ there exists a $\sigma$-additive probability measure $\mu_{c}$ supported on the set $\operatorname{ext}(C)$ of extreme points of $C$ such that

$$
a(c)=\int_{\operatorname{ext}(C)} a(e) \mathrm{d} \mu_{c}(e) \text { for all } a \in A(C) .
$$

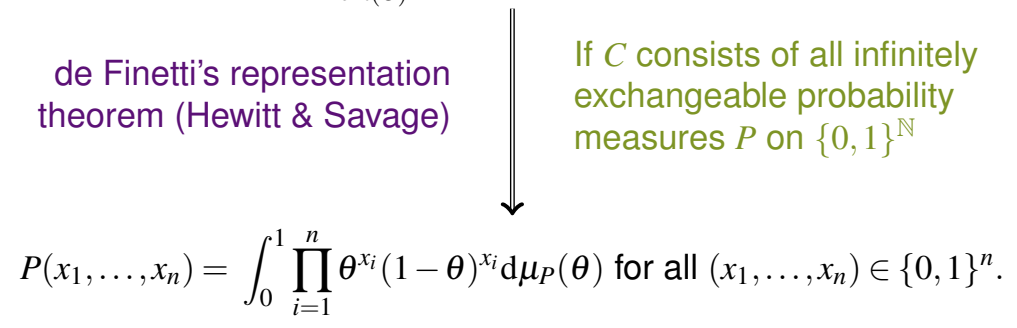




\section{Bishop-De Leeuw theorem}

Let $V$ be a locally convex Hausdorff topological linear space, and let $C$ be a non-empty convex and compact subset of $V$. Denote by $A(C)$ the linear space of all continuous affine real maps $a$ on $C$. Then for every $c \in C$ there exists a $\sigma$-additive probability measure $\mu_{c}$ supported on the set $\operatorname{ext}(C)$ of extreme points of $C$ such that

$$
a(c)=\int_{\operatorname{ext}(C)} a(e) \mathrm{d} \mu_{c}(e) \text { for all } a \in A(C) .
$$

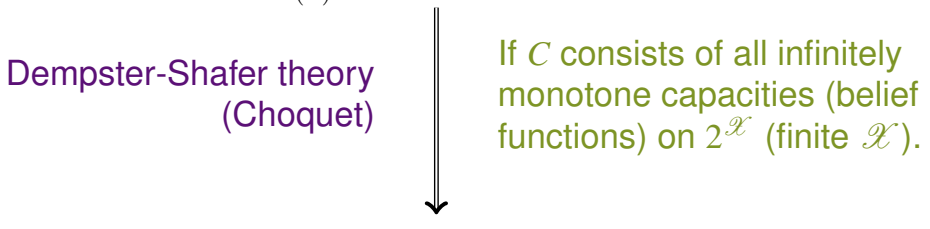

$$
\operatorname{bel}(A)=\sum_{B \subseteq A} m(B)=\sum_{B \subseteq \mathscr{X}} \operatorname{bel}_{B}(A) m(B) \text { for all } A \subseteq \mathscr{X} .
$$




\section{Bishop-De Leeuw theorem}

Let $V$ be a locally convex Hausdorff topological linear space, and let $C$ be a non-empty convex and compact subset of $V$. Denote by $A(C)$ the linear space of all continuous affine real maps $a$ on $C$. Then for every $c \in C$ there exists a $\sigma$-additive probability measure $\mu_{c}$ supported on the set $\operatorname{ext}(C)$ of extreme points of $C$ such that

$$
a(c)=\int_{\operatorname{ext}(C)} a(e) \mathrm{d} \mu_{c}(e) \text { for all } a \in A(C) .
$$

Ph.D. thesis Maaß

(Troffaes \& De Cooman)

If $C$ is a set of so-called 'inequality preserving' functionals $c$.

$$
c(\omega)=\int_{\operatorname{ext}(C)} e(\omega) \mathrm{d} \mu_{c}(e) \text { for all } \omega \in \Omega .
$$




\section{Bishop-De Leeuw theorem}

Let $V$ be a locally convex Hausdorff topological linear space, and let $C$ be a non-empty convex and compact subset of $V$. Denote by $A(C)$ the linear space of all continuous affine real maps $a$ on $C$. Then for every $c \in C$ there exists a $\sigma$-additive probability measure $\mu_{c}$ supported on the set $\operatorname{ext}(C)$ of extreme points of $C$ such that

$$
a(c)=\int_{\operatorname{ext}(C)} a(e) \mathrm{d} \mu_{c}(e) \text { for all } a \in A(C) .
$$

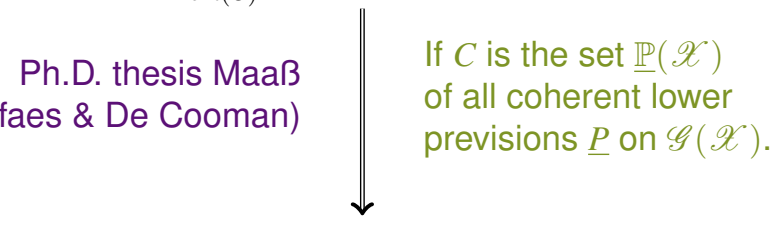

$$
\underline{P}(f)=\int_{\operatorname{ext} \underline{\mathbb{P}}(\mathscr{X})} \underline{P}_{\text {ext }}(f) \mathrm{d} \mu_{\underline{P}}\left(\underline{P}_{\text {ext }}\right) \text { for all } f \in \mathscr{G}(\mathscr{X}) .
$$




\section{Bishop-De Leeuw theorem}

Let $V$ be a locally convex Hausdorff topological linear space, and let $C$ be a non-empty convex and compact subset of $V$. Denote by $A(C)$ the linear space of all continuous affine real maps $a$ on $C$. Then for every $c \in C$ there exists a $\sigma$-additive probability measure $\mu_{c}$ supported on the set $\operatorname{ext}(C)$ of extreme points of $C$ such that

$$
a(c)=\int_{\operatorname{ext}(C)} a(e) \mathrm{d} \mu_{c}(e) \text { for all } a \in A(C) .
$$

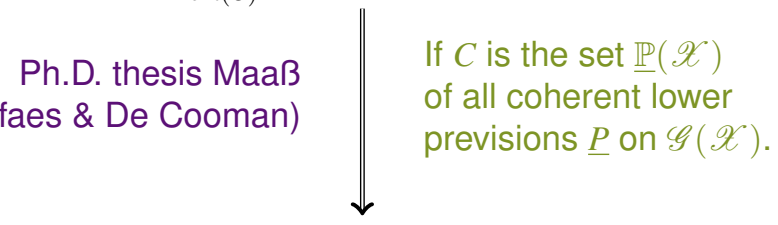

$$
\underline{P}(f)=\int_{\operatorname{ext} \underline{\underline{P}}(\mathscr{X})} \underline{P}_{\mathrm{ext}}(f) \mathrm{d} \mu_{\underline{P}}\left(\underline{P}_{\mathrm{ext}}\right) \text { for all } f \in \mathscr{G}(\mathscr{X}) .
$$




\section{Coherent lower previsions}

A variable $X$ takes values $x$ in some non-empty $\mathscr{X}$ (finite in this talk).

$\mathscr{G}(\mathscr{X})$ is the set of all gambles $f$ (real valued maps) on $\mathscr{X}$.

A coherent lower prevision $\underline{P}$ is a real valued functional on $\mathscr{G}(\mathscr{X})$ such that for all $f, g \in \mathscr{G}(\mathscr{X})$ and all real $\lambda>0$

$$
\begin{aligned}
& \text { 1. } \underline{P}(f) \geq \min f \\
& \text { 2. } \underline{P}(\lambda f)=\lambda \underline{P}(f) \\
& \text { 3. } \underline{P}(f+g) \geq \underline{P}(f)+\underline{P}(g)
\end{aligned}
$$

[boundedness] [non-negative homogeneity] [super-additivity]

We denote the set of all coherent lower previsions on $\mathscr{G}(\mathscr{X})$ by $\mathbb{P}(\mathscr{X})$. 


\section{Coherent lower previsions}

A variable $X$ takes values $x$ in some non-empty $\mathscr{X}$ (finite in this talk).

$\mathscr{G}(\mathscr{X})$ is the set of all gambles $f$ (real valued maps) on $\mathscr{X}$.

A coherent lower prevision $\underline{P}$ is a real valued functional on $\mathscr{G}(\mathscr{X})$ such that for all $f, g \in \mathscr{G}(\mathscr{X})$ and all real $\lambda>0$

$$
\begin{aligned}
& \text { 1. } \underline{P}(f) \geq \min f \\
& \text { 2. } \underline{P}(\lambda f)=\lambda \underline{P}(f) \\
& \text { 3. } \underline{P}(f+g) \geq \underline{P}(f)+\underline{P}(g)
\end{aligned}
$$

[boundedness] [non-negative homogeneity] [super-additivity]

We denote the set of all coherent lower previsions on $\mathscr{G}(\mathscr{X})$ by $\mathbb{P}(\mathscr{X})$.

There is a one-to-one correspondence with credal sets:

$$
\begin{aligned}
\mathscr{M}_{\underline{P}} & =\left\{p \in \Sigma_{\mathscr{X}}: P_{p}(f) \geq \underline{P}(f) \text { for all } f \in \mathscr{G}(\mathscr{X})\right\}, \\
\underline{P}(f) & =\min \left\{P_{p}(f): p \in \mathscr{M}_{\underline{P}}\right\} \text { for all } f \in \mathscr{G}(\mathscr{X}) .
\end{aligned}
$$

We denote the set of all credal sets on $\mathscr{X}$ by $\mathbb{M}(\mathscr{X})$. 


\section{Coherent lower previsions}

We partition $\mathbb{P}(\mathscr{X})$ in three disjoint subsets

1. $\mathbb{P}(\mathscr{X})$ : the set of all linear previsions

2. $\mathbb{P}(\mathscr{X})$ : the set of all fully imprecise lower previsions

3. $\underset{\sim}{\mathbb{P}}(\mathscr{X})$ : the set of all partially imprecise lower previsions 


\section{Coherent lower previsions}

We partition $\mathbb{P}(\mathscr{X})$ in three disjoint subsets

1. $\mathbb{P}(\mathscr{X})$ : the set of all linear previsions

2. $\mathbb{P}(\mathscr{X})$ : the set of all fully imprecise lower previsions

3. $\underset{\sim}{\mathbb{P}}(\mathscr{X})$ : the set of all partially imprecise lower previsions

Linear previsions

$$
\underline{P} \in \mathbb{P}(\mathscr{X}) \Longleftrightarrow \mathscr{M}_{\underline{P}}=\{p\} \text {, with } p \in \Sigma_{\mathscr{X}}
$$

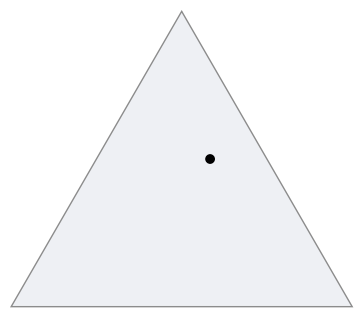




\section{Coherent lower previsions}

We partition $\mathbb{P}(\mathscr{X})$ in three disjoint subsets

1. $\mathbb{P}(\mathscr{X})$ : the set of all linear previsions

2. $\mathbb{P}(\mathscr{X})$ : the set of all fully imprecise lower previsions

3. $\underset{\sim}{\mathbb{P}}(\mathscr{X})$ : the set of all partially imprecise lower previsions

Fully imprecise lower previsions

$$
\underline{P} \in \underline{\underline{P}}(\mathscr{X}) \Longleftrightarrow\left(\forall i \in \mathbb{N}_{\leq n}\right) \underline{P}\left(\mathbb{I}_{\left\{x_{i}\right\}}\right)=0
$$

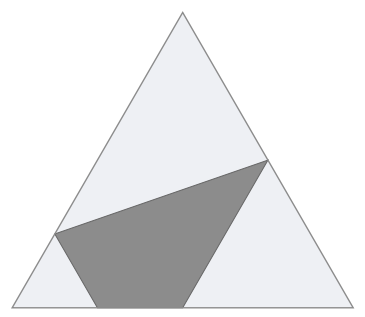




\section{Coherent lower previsions}

We partition $\mathbb{P}(\mathscr{X})$ in three disjoint subsets

1. $\mathbb{P}(\mathscr{X})$ : the set of all linear previsions

2. $\mathbb{P}(\mathscr{X})$ : the set of all fully imprecise lower previsions

3. $\underset{\sim}{\mathbb{P}}(\mathscr{X})$ : the set of all partially imprecise lower previsions

Partially imprecise lower previsions

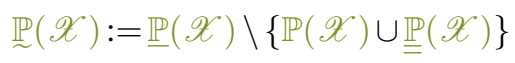

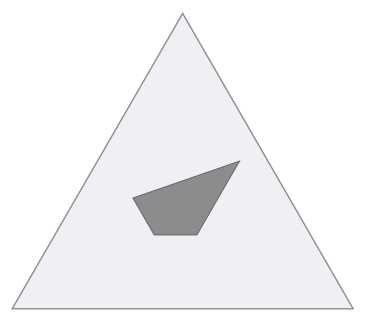




\section{Extreme lower previsions}

A coherent lower prevision $\underline{P} \in \mathbb{P}(\mathscr{X})$ is called extreme if it is not possible to find $\underline{P}_{1}$ and $\underline{P}_{2}$ in $\underline{\mathbb{P}}(\mathscr{X})$, with $\underline{P}_{1} \neq \underline{P}_{2}$, and $\lambda \in(0,1)$ such that $\underline{P}=\lambda \underline{P}_{1}+(1-\lambda) \underline{P}_{2}$, meaning that

$$
\underline{P}(f)=\lambda \underline{P}_{1}(f)+(1-\lambda) \underline{P}_{2}(f) \text { for all } f \in \mathscr{G}(\mathscr{X}) .
$$

What is the set $\operatorname{ext} \mathbb{P}(\mathscr{X})$ of all extreme lower previsions on $\mathscr{G}(\mathscr{X})$ ? 


\section{Extreme lower previsions}

A coherent lower prevision $\underline{P} \in \mathbb{P}(\mathscr{X})$ is called extreme if it is not possible to find $\underline{P}_{1}$ and $\underline{P}_{2}$ in $\underline{P}(\mathscr{X})$, with $\underline{P}_{1} \neq \underline{P}_{2}$, and $\lambda \in(0,1)$ such that $\underline{P}=\lambda \underline{P}_{1}+(1-\lambda) \underline{P}_{2}$, meaning that

$$
\underline{P}(f)=\lambda \underline{P}_{1}(f)+(1-\lambda) \underline{P}_{2}(f) \text { for all } f \in \mathscr{G}(\mathscr{X}) .
$$

What is the set $\operatorname{ext} \mathbb{P}(\mathscr{X})$ of all extreme lower previsions on $\mathscr{G}(\mathscr{X})$ ?

A credal set $\mathscr{M} \in \mathbb{M}(\mathscr{X})$ is called extreme if it is not possible to find $\mathscr{M}_{1}$ and $\mathscr{M}_{2}$ in $\mathbb{M}(\mathscr{X})$, with $\mathscr{M}_{1} \neq \mathscr{M}_{2}$, and $\lambda \in(0,1)$ such that $\mathscr{M}=\lambda \mathscr{M}_{1}+(1-\lambda) \mathscr{M}_{2}$, meaning that

$$
\mathscr{M}=\left\{\lambda p_{1}+(1-\lambda) p_{2}: p_{1} \in \mathscr{M}_{1} \text { and } p_{2} \in \mathscr{M}_{2}\right\} .
$$

What is the set ext $\mathbb{M}(\mathscr{X})$ of all extreme credal sets on $\mathscr{X}$ ? 


\section{Extreme lower previsions}

A coherent lower prevision $\underline{P} \in \mathbb{P}(\mathscr{X})$ is called extreme if it is not possible to find $\underline{P}_{1}$ and $\underline{P}_{2}$ in $\underline{P}(\mathscr{X})$, with $\underline{P}_{1} \neq \underline{P}_{2}$, and $\lambda \in(0,1)$ such that $\underline{P}=\lambda \underline{P}_{1}+(1-\lambda) \underline{P}_{2}$, meaning that

$$
\underline{P}(f)=\lambda \underline{P}_{1}(f)+(1-\lambda) \underline{P}_{2}(f) \text { for all } f \in \mathscr{G}(\mathscr{X}) .
$$

What is the set $\operatorname{ext} \mathbb{P}(\mathscr{X})$ of all extreme lower previsions on $\mathscr{G}(\mathscr{X})$ ?

$$
\underline{P} \in \operatorname{ext} \underline{\mathbb{P}}(\mathscr{X}) \Leftrightarrow \mathscr{M}_{\underline{P}} \in \operatorname{ext} \underline{\mathbb{M}}(\mathscr{X})
$$

A credal set $\mathscr{M} \in \mathbb{M}(\mathscr{X})$ is called extreme if it is not possible to find $\mathscr{M}_{1}$ and $\mathscr{M}_{2}$ in $\mathbb{M}(\mathscr{X})$, with $\mathscr{M}_{1} \neq \mathscr{M}_{2}$, and $\lambda \in(0,1)$ such that $\mathscr{M}=\lambda \mathscr{M}_{1}+(1-\lambda) \mathscr{M}_{2}$, meaning that

$$
\mathscr{M}=\left\{\lambda p_{1}+(1-\lambda) p_{2}: p_{1} \in \mathscr{M}_{1} \text { and } p_{2} \in \mathscr{M}_{2}\right\} .
$$

What is the set ext $\mathbb{M}(\mathscr{X})$ of all extreme credal sets on $\mathscr{X}$ ? 


\section{Extreme lower previsions are never partially imprecise}

Any partially imprecise lower prevision $\underline{P} \in \mathbb{P}(\mathscr{X})$ can be uniquely written as a convex combination $\lambda P_{1}+(1-\lambda) \underline{P}_{2}$ of a linear prevision $P_{1} \in \mathbb{P}(\mathscr{X})$ and a fully imprecise lower prevision $\underline{P}_{2} \in \underline{\underline{P}}(\mathscr{X})$.

$$
\begin{aligned}
& P_{1}(f)=\frac{1}{\lambda} \sum_{i=1}^{n} f\left(x_{i}\right) \underline{P}\left(\mathbb{I}_{\left\{x_{i}\right\}}\right) \text { for all } f \in \mathscr{G}(\mathscr{X}) \\
& \underline{P}_{2}(f)=\frac{1}{1-\lambda} \underline{P}(f)-\frac{\lambda}{1-\lambda} P_{1}(f) \text { for all } f \in \mathscr{G}(\mathscr{X})
\end{aligned} \quad \lambda=\sum_{i=1}^{n} \underline{P}\left(\mathbb{I}_{\left\{x_{i}\right\}}\right)
$$
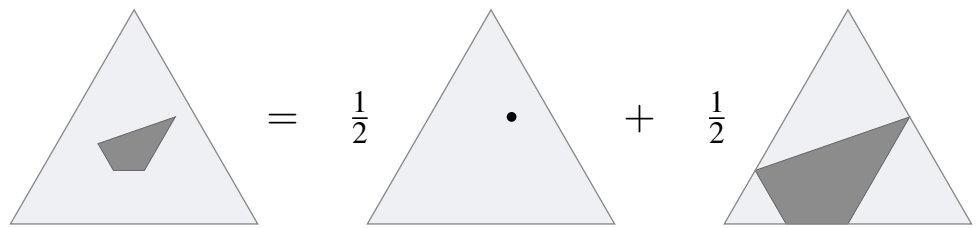


\section{Extreme lower previsions: the linear ones}

A linear prevision $P \in \mathbb{P}(\mathscr{X})$ is extreme if and only if it is degenerate. Furthermore, any other linear prevision can be uniquely written as a convex combination of these degenerate ones.

$$
\begin{aligned}
& P(f)=\sum_{i=1}^{n} p\left(x_{i}\right) P_{i}^{\circ}(f) \text { for all } f \in \mathscr{G}(\mathscr{X}) \\
& P_{i}^{\circ}(f):=f\left(x_{i}\right) \text { for all } f \in \mathscr{G}(\mathscr{X})
\end{aligned}
$$

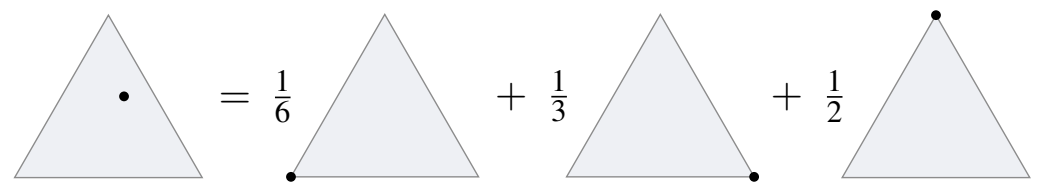




\section{Extreme lower previsions: the fully imprecise ones}

A fully imprecise lower prevision $\underline{P} \in \mathbb{P}(\mathscr{X})$ is extreme if and only if its projected credal set $K_{\underline{P}}$ is Minkowski indecomposable.
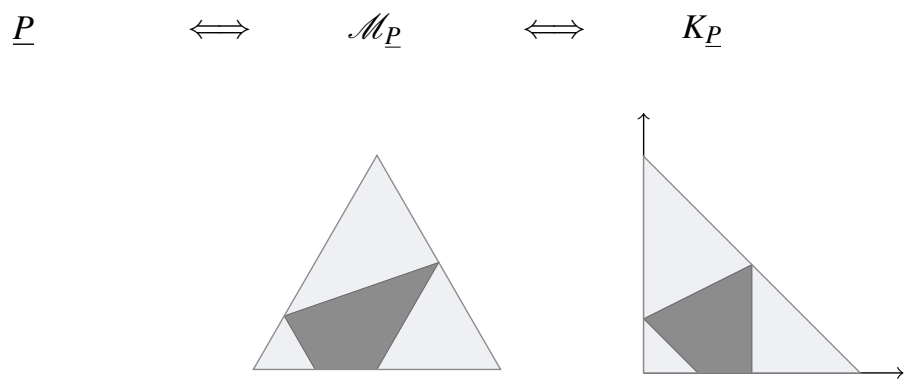


\title{
Minkowski decomposition
}
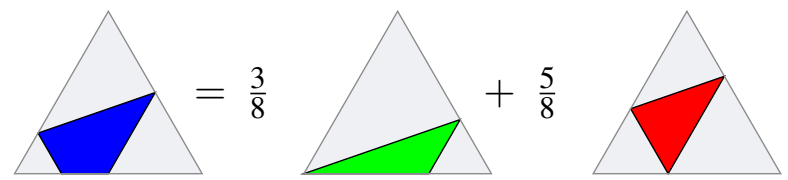

\author{
credal \\ decomposition
}
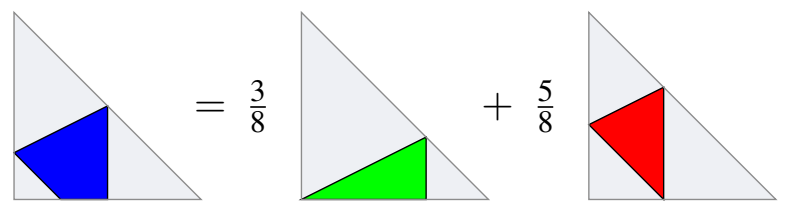

\author{
projected \\ credal \\ decomposition
}
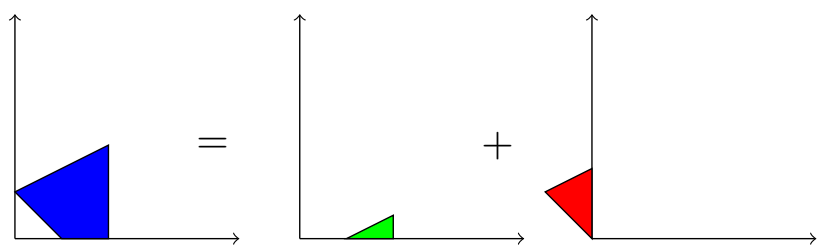

\author{
Minkowski \\ decomposition
}




\section{Extreme lower previsions: $n=1, \mathscr{X}=\{x\}$}

A variable that can assume only one value has no uncertainty associated with it... 


\section{Extreme lower previsions: $n=2, \mathscr{X}=\left\{x_{1}, x_{2}\right\}$}

The linear extreme lower previsions are the degenerate ones.
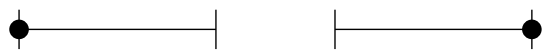

There is only one fully imprecise extreme lower prevision.

There are no partially imprecise extreme lower previsions.

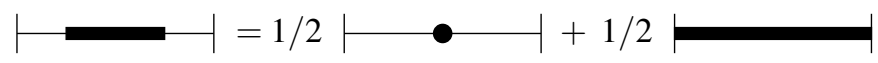




\section{Extreme lower previsions: $n=3, \mathscr{X}=\left\{x_{1}, x_{2}, x_{3}\right\}$}

Silverman, R. Decomposition of plane convex sets, part I. Pacific Journal of Mathematics 47, 521-530 (1973)

For possibility spaces $\mathscr{X}=\left\{x_{1}, x_{2}, x_{3}\right\}$ containing only three elements, a fully imprecise lower prevision $\underline{P} \in \mathbb{M}(\mathscr{X})$ is extreme if and only if it is the lower envelope of three linear previsions.
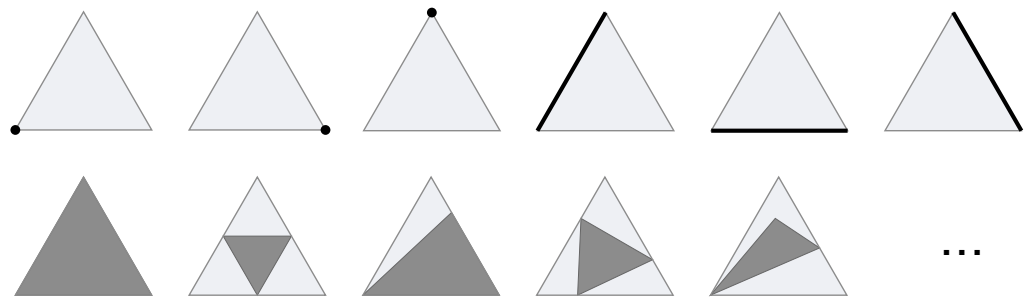


\section{Extreme lower previsions: $n>3$}

It gets rather more complicated! However, quite a lot of results are available... We refer to the literature on Minkowski decomposition.

Grünbaum, B.

Convex polytopes.

Springer, 2nd edition (2003)

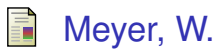

Indecomposable polytopes.

Transactions of the American Mathematical Society 190, 77-86

(1974)

Sallee, G.T.

Minkowski decomposition of convex sets.

Israel Journal of Mathematics 12, 266-276 (1972) 


\section{Decomposing non-extreme lower previsions}

Decomposition of a partially imprecise lower prevision, $n=3$
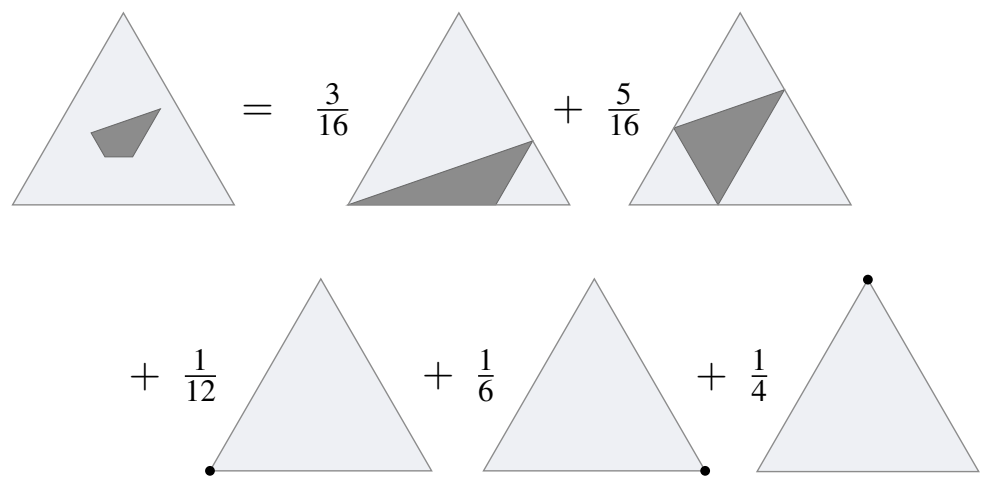


\section{Decomposing non-extreme lower previsions}

Decomposition of a fully imprecise lower prevision, $n=3$

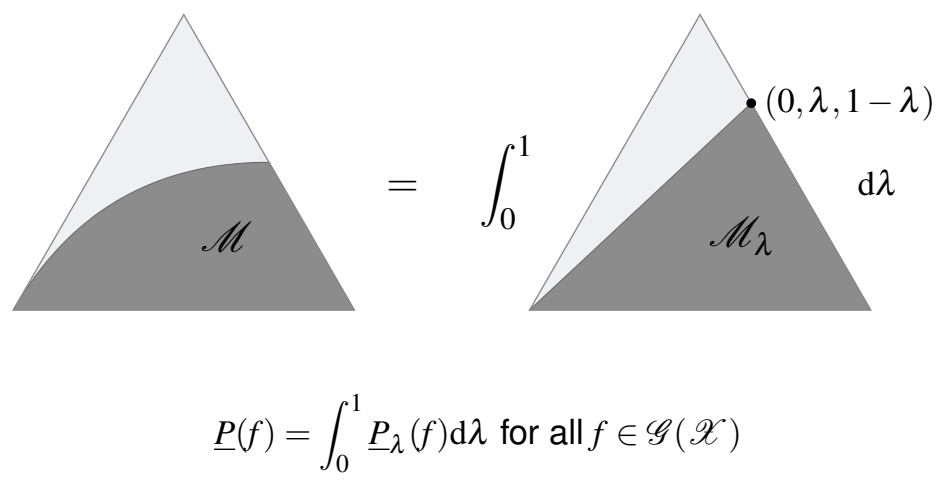




\section{Monte Carlo with imprecise probabilities!}

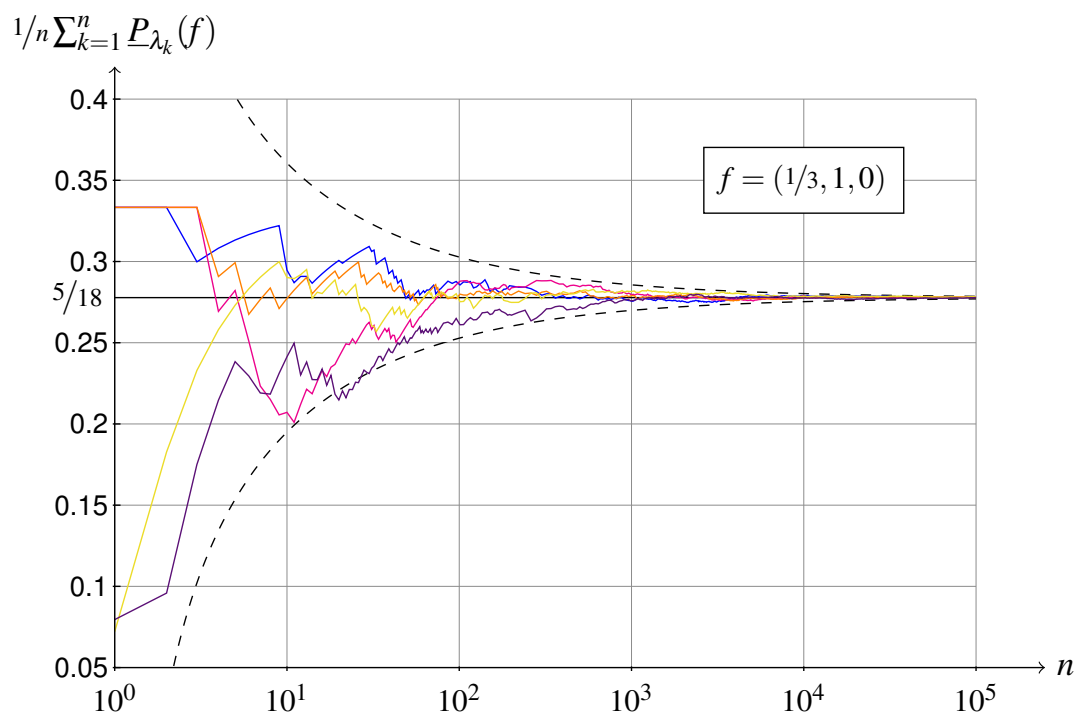




\section{Further reading}

Coherent lower previsions

凬 Miranda, E.

A survey of the theory of coherent lower previsions.

International Journal of Approximate Reasoning 48(2), 628-658 (2008)

诖 Walley, P.

Statistical Reasoning with Imprecise Probabilities.

Chapman and Hall, London (1991)

园 Troffaes, M., De Cooman, G.

Lower Previsions.

Wiley \& Sons (soon to be published!) 


\section{Further reading}

Representation results, extreme lower previsions, ...

Maaß, S.

Exact functionals, functionals preserving linear inequalities,

Lévy's metric.

Ph.D. thesis, Universität Bremen (2003)

葍 Quaeghebeur, E.

Characterizing the set of coherent lower previsions with a finite number of constraints or vertices.

Proceedings of UAI 2010, 466-473 (2010)

囯 Quaeghebeur, E., De Cooman, G.

Extreme lower probabilities.

Fuzzy Sets and Systems 159, 2163-2175 (2008) 


\section{Further reading}

Minkowski decomposition

Grünbaum, B.

Convex polytopes.

Springer, 2nd edition (2003)

酒 Meyer, W.

Indecomposable polytopes.

Transactions of the American Mathematical Society 190, 77-86

(1974)

言 Sallee, G.T.

Minkowski decomposition of convex sets.

Israel Journal of Mathematics 12, 266-276 (1972)

Silverman, R.

Decomposition of plane convex sets, part I.

Pacific Journal of Mathematics 47, 521-530 (1973) 